\title{
The distribution of psychosis, ICD-11 PTSD and complex PTSD symptoms among a trauma- exposed UK general population sample
}

\section{Rachel Frost, Maria Louison Vang, Thanos Karatzias, Philip Hyland \& Mark} Shevlin

To cite this article: Rachel Frost, Maria Louison Vang, Thanos Karatzias, Philip Hyland \& Mark Shevlin (2019) The distribution of psychosis, ICD-11 PTSD and complex PTSD symptoms among a trauma-exposed UK general population sample, Psychosis, 11:3, 187-198, DOI: 10.1080/17522439.2019.1626472

To link to this article: https://doi.org/10.1080/17522439.2019.1626472

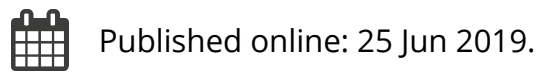

Submit your article to this journal $\widetilde{ }$

Lll Article views: 509

Q View related articles $\sqsubset$

View Crossmark data $[\pi$

Citing articles: 2 View citing articles \ulcorner 


\title{
The distribution of psychosis, ICD-11 PTSD and complex PTSD symptoms among a trauma-exposed UK general population sample
}

\author{
Rachel Frost (D) $^{a}$, Maria Louison Vang (iD) ${ }^{a}$, Thanos Karatzias (iD ${ }^{b}$, Philip Hyland (iD ${ }^{c}$ \\ and Mark Shevlin (iD) \\ aSchool of Psychology, Ulster University, Coleraine Campus, Northern Ireland; bepartment of Psychology, \\ Edinburgh Napier University, Edinburgh, UK; 'School of Health \& Social Care, National University of Ireland \\ Maynooth, Republic of Ireland
}

\begin{abstract}
Background: Co-occurrence of psychosis and posttraumatic stress disorder (PTSD) symptoms has been demonstrated, but the ICD-11 marks a significant divergence in the formulation of PTSD with a focus on the core symptoms and the addition of complex PTSD (CPTSD).

Objective: To evaluate the distribution of psychosis and traumatic stress symptoms using the ICD-11 conceptualisation of PTSD and CPTSD.

Method: A latent class analysis was conducted on psychosis symptoms, PTSD and CPTSD among a random adult sample from the UK general population with a history of traumatic events $(\mathrm{N}=1,051)$.

Results: Six classes were identified; a low-symptom class, a PTSD-class, a CPTSD-class, a class characterized by disturbances in self-organization alone as well as two classes characterized by CPTSD and various levels of psychosis symptom endorsement. Cumulative childhood adversity predicted membership of the PTSD, CPTSD and comorbid classes in a dose-response manner with the strongest effects observed for classes characterised by comorbid symptoms.

Conclusion: The present study confirms the co-occurrence of psychosis symptoms and ICD-11 PTSD and CPTSD. Psychosis symptoms did not emerge in isolation from traumatic stress symptoms, underpinning the need for a greater recognition of psychosis symptoms as part of the broader clinical picture among trauma-exposed populations.
\end{abstract}

\section{ARTICLE HISTORY}

Received 21 March 2019

Accepted 29 May 2019

\section{KEYWORDS}

Complex PTSD; PTSD;

psychosis; childhood trauma

\section{Introduction}

The International Classification of Diseases 11th edition manual (ICD-11) outlines two distinct but trauma related conditions, marking a significant divergence from the Diagnostic and Statistical Manual in terms of the formulation of posttraumatic stress disorder (PTSD) with a focus on the core symptoms and the addition of complex PTSD (CPTSD) as a new diagnosis (Maercker et al., 2013). In ICD-11, PTSD was refined to six symptoms, subsumed under three symptoms clusters; (i) reexperiencing, (ii) avoidance, and (iii) sense of threat (Maercker et al., 2013). CPTSD is comprised of both the core PTSD symptoms plus three additional symptom clusters collectively referred to as "Disturbance in Self Organisation" (DSO); (i) affective dysregulation, (ii) negative-self-concept, and (iii) disturbed relationships. An accumulating body of evidence documents the validity of ICD-11 PTSD and CPTSD (Brewin et al., 2017; Cloitre et al., 2018). Similarly, research has documented a recurrent link between trauma-exposure, especially during early development, and psychotic disorders or psychotic-like experiences (Bailey et al., 2018; Gibson, Alloy, \& Ellman, 2016). Despite 
this evidence, concern remains that trauma-history and traumatic stress go underrecognized among individuals with a psychosis (Hardy \& Mueser, 2017; Read, van Os, Morrison, \& Ross, 2005).

Psychosis and PTSD have previously been conceptualised as similar entities that lie on a spectrum of reactions to trauma (Morrison, Frame, \& Larkin, 2003). This view emphasises the phenomenological similarity of flashbacks and hallucinations as unwelcome intrusions of unpleasant situations that are not happening in the here-and-now despite their subjective vividness. Consequently, it is proposed that the labelling of intrusive experiences as "delusions" and "hallucinations" or "intrusions" and "flashbacks" determines the diagnostic interpretation of these symptoms as either a function of psychosis or PTSD (Morrison et al., 2003; Morrison, Read, \& Turkington, 2005). Additionally, individuals diagnosed with a psychotic disorder report a higher prevalence of PTSD compared to the general population, although comorbidity rates vary depending on the population sampled and methodology employed (Achim et al., 2009; Kessler et al., 2005).

Investigating the overlap between PTSD and psychosis in terms of comorbidity-rates imposes a separation of symptoms that might be a suboptimal representation if these syndromes are to be understood as different "points" on an underlying spectrum of trauma-related reactions (Morrison et al., 2003). Rather than imposing an a priori separation of the disorders predicated on diagnostic criteria, Shevlin, Armour, Murphy, Houston, and Adamson (2011) modelled the co-occurrence of symptoms of PTSD and psychosis using latent class analysis (LCA) among a US community sample with a lifetime PTSD diagnosis. LCA tests for homogenous groups of individuals (i.e. "classes") that can be objectively identified based on patterns of symptom-endorsement. Shevlin et al. (2011) identified four classes: all classes had a moderate to high probability of endorsing PTSD symptoms, and two of these classes were additionally characterized by endorsement of psychosis symptoms, particularly hallucinations and delusions. Membership of classes characterized by psychosis symptoms was predicted by trauma exposure and associated with elevated levels of clinical comorbidity. These findings highlight the importance of recognising symptoms that lie beyond the diagnostic boundaries of PTSD when assessing posttraumatic sequelae. The importance of this is further underlined by the shared risk-factors of psychosis, PTSD and, most recently, CPTSD.

Childhood adversity poses a key etiological risk factor in the development of psychosis (Arseneault et al., 2011; Varese et al., 2012). This relationship persists despite controlling for potential confounds such as genetic risk, family psychiatric history, comorbid psychopathology, cannabis use, ethnicity, urbanicity and educational attainment (Houston, Murphy, Adamson, Stringer, \& Shevlin, 2008; Husted, Ahmed, Chow, Brzustowicz, \& Bassett, 2010; Shevlin, Houston, Dorahy, \& Adamson, 2008). A strong dose-response effect has been evidenced between childhood adversity and psychosis, such that the relative risk of psychosis disorder or symptom increases for each additional trauma reported (Shevlin et al., 2008; Trauelsen et al., 2015), and individuals with a psychotic disorder and a history of childhood adversity present with higher rates of psychiatric co-morbidity alike other populations with a childhood trauma history (Schäfer \& Fisher, 2011).

Similarly, childhood adversity is one of the most well-established predictors of ICD-11 PTSD and CPTSD (Cloitre, Garvert, Brewin, Bryant, \& Maercker, 2013; Karatzias et al., 2017). CPTSD was originally proposed in ICD-11 to capture the greater variety and severity of traumatic stress responses that emerge following sustained or repeated trauma exposure, especially that of an interpersonal nature, and especially victimizations that occur early in development (Herman, 1992; Maercker et al., 2013). Considering the revisions of disorders related to traumatic stress in ICD-11, more research is required to explore the relationship between traumatic stress and psychotic symptoms, particularly the relationship between CPTSD and psychosis symptoms.

The aim of this study is to evaluate the association between trauma exposure, psychosis symptoms, PTSD and DSO symptoms based on the recent ICD-11 revisions. LCA was employed, and based on previous findings (Shevlin et al., 2011), we predicted that the LCA would identify classes characterised by, but not limited to; (1) a PTSD symptom profile; (2) a CPTSD symptom profile and (3) a profile characterized by overlapping traumatic stress symptoms and psychosis 
symptoms. Secondly, we assessed if individual types of childhood adversity and cumulative childhood adversity discriminated between the resultant classes. Lastly, we investigated if classes could be differentiated by level of comorbid psychopathology.

\section{Method}

\section{Sample}

An adult sample of the UK population was selected from an existing online research panel that was developed to be representative of the adult UK population. An aggregated panel of respondents who's GEO-IP address was based in the UK were randomly recruited through probability-based sampling in order to recruit approximately 1,000 participants. Email invitations were sent out in waves and the take-up rate was monitored in field. Respondents then went through a prescreening criteria check where they were asked about their age and gender. This is done to meet the quota to approximate a representative sample. This enabled the first 2 inclusion criteria for sample recruitment to be met: (a) be born in the UK and (b) be aged 18 years or older at the time of the survey. If these criteria were met the third inclusion criteria, screening positive for at least one traumatic event in their lifetime, was applied using the Life Events Checklist (LEC). Participants who endorsed any item on the LEC then completed the remaining battery of measures in the survey. In total 2,653 panel members were contacted and 1,051 people qualified for inclusion in the final analyses (selection rate $=39.6 \%$ ). Ethical approval for the collection of data from this sample was granted by the ethical review board of the institution to which one of the authors is affiliated. No inducements or incentives were offered for participation.

Most of the sample was female $(n=719,68.4 \%)$ with a mean age of 47.18 years (SD $=15.00$, range $=18-90$ years). The majority of respondents had completed a college or university education $(62.7 \%, \mathrm{n}=659)$, were in full or part time employment $(58.5 \%, \mathrm{n}=615)$, were "in a committed relationship" $(70.4 \%, \mathrm{n}=740)$ and had no children under the age of 16 years $(67.5 \%, \mathrm{n}=709)$. A minority indicated that they had emigrated at some point $(17.8 \%, n=187)$.

\section{Measures}

\section{PTSD and CPTSD}

The International Trauma Questionnaire (ITQ; Cloitre et al., 2018) was used to assess PTSD and CPTSD. The PTSD subscale consists of 6 items assessing re-experiencing (2 items), avoidance (2 items) and sense of threat ( 2 items). The disturbances in self-organization (DSO) subscale consists of 6 items assessing the occurrence of affective dysregulation (2 items), negative self-concept (2 items) and disturbances in interpersonal relationships (2 items). All items are scored on a 5-point Likert scale ranging from "Not at all (0)" to "Extremely (4)". In line with recommendations, an item is considered endorsed when respondents indicate a score of 2 ("Moderately") or more. According to ICD-11 diagnostic criteria, a symptom is considered present if at least one of the two items from the respective cluster has been endorsed. The reliability of the total scale $(a=0.93)$, the PTSDsubscale $(a=0.91)$ and CPTSD subscale $(\alpha=0.92)$ was excellent.

\section{Psychosis symptoms}

A modified version of the Adolescent Psychotic-like Symptom Screener (APSS; Kelleher, Harley, Murtagh, \& Cannon, 2011) was used to measure psychotic symptoms. This is a 7 item self-report questionnaire where participants are instructed to indicate if they had ever had the experience in question, and if so, whether this experience had caused them any distress. The items were:

(1) Some people believe that their thoughts can be read by another person. Have other people ever read your mind? (mind reading)

(2) Have you ever had messages sent just to you through the TV or radio? (special messages) 
(3) Have you ever thought that people are following or spying on you? (spying on you)

(4) Have you ever heard voices or sounds that no one else can hear? (auditory hallucinations)

(5) Have you ever felt you were under the control of some special power? (under control)

(6) Have you ever seen things that other people could not see? (visual hallucinations)

(7) Have you ever felt like you had extra-special powers? (special powers)

Items were rated on a four-point Likert scale: "Never", "Sometimes", "Often", and "Nearly Always". An item was considered to indicate a "psychotic experience" if "Sometimes", "Often", or "Nearly Always" was endorsed. If an item was endorsed, participants were asked to rate how distressing it was on a four-point Likert scale: "Not distressed", "A bit distressed", "Quite distressed", and "Very distressed". Each item was considered to reflect a "psychosis symptom" if distress related to an experience was rated as "A bit distressed", "Quite distressed", or "Very distressed". Kelleher et al. (2011) reported that APSS scores detected adolescents with clinical interview verified psychotic experiences with a sensitivity of $70 \%$ and a specificity of $82.6 \%$. Nolan et al. (2018) found that APSS frequency and distress scores differentiated adolescent community participants from adult participants with a history of sexual trauma and another group of participants with serious mental health problems using supported living services.

\section{Childhood adversity}

Childhood adversity was assessed using the Adverse Childhood Experiences (ACE; Felitti et al., 1998). The ACE is a 10-item self-report measure items are scored in a "Yes" (1) and "No" (0) response format. Five items were selected from the ACE to measure emotional neglect, physical neglect, verbal abuse, sexual abuse and physical abuse.

\section{Adult trauma}

Adult trauma was assessed using a modified version of the Life-Events Checklist (LEC: Weathers et al., 2013b). The original LEC is a 17-item self-report screening-tool for lifetime exposure to 16 potentially traumatizing events, and the response format was simplified to use only a "Yes" (1) and "No" (0) response format. The list of events was prefixed with, "Happened in Adulthood (at or after age 18)" to capture experiences in adulthood. One open-ended question was included for respondents to indicate any other traumatic event not listed (Weathers et al., 2013b).

\section{Anxiety}

The Generalized Anxiety Disorder-7 (GAD-7; Spitzer, Kroenke, Williams, \& Löwe, 2006) was used to assess symptoms of anxiety. The GAD-7 is scored on a 4-point Likert scale from "Not at all" (0) to "nearly every day" (3). Higher total scores indicate higher severity of anxiety with a cut-off score of 10 and 15 indicating moderate and severe levels of anxiety respectively. The internal reliability of the scale was excellent $(\alpha=.95)$.

\section{Depression}

The Patient Health Questionnaire-9 (PHQ-9; Kroenke, Spitzer, \& Williams, 2001) was used to assess symptoms of depression. The PHQ-9 is a 9-item self-report measure, higher scores indicate higher severity with a cut-off of 15 indicating levels of depressive symptomatology that warrants treatment. The internal reliability of the scale was excellent $(a=0.94)$.

\section{Cannabis use}

Cannabis use was assessed using a single item, "Have you ever smoked/used cannabis?" which used a "Yes" (1) and "No" (0) response format. 


\section{Analysis}

First, symptom-endorsement was computed for the ITQ and the APSS and a latent class analysis (LCA) was conducted to determine symptom profiles across PTSD, DSO and psychosis symptoms. The "symptom" scoring for the APSS was used where item endorsement required a frequency rating greater than "Never" and distress rating greater than "Not distressed". LCA was used to uncover distinct groups of individuals based on patterns of symptom endorsement (presence or absence) of PTSD, DSO and psychosis symptoms. The fit of eight models (a 1-class through 8-class model) was assessed. The model parameters were estimated using robust maximum likelihood and to avoid solutions based on local maxima 5000 random sets of starting values were used initially and 1000 final stage optimizations (Yuan \& Bentler, 2000). To identify the optimal class solution the following fit statistics were compared: the Akaike Information Criterion (AIC; Akaike, 1998), the Bayesian Information Criterion (BIC; Schwarz, 1978) and the sample size-adjusted Bayesian Information Criterion (ssaBIC; Sclove, 1987). Lower value indicate good model fit, with evidence to suggest that the BIC is the best fitting information criterion for identifying the optimal class solution (Nylund, Asparouhov, \& Muthén, 2007). Entropy values were additionally examined to discriminate between class solutions, with values above .8 indicating acceptable classification. The Lo-Mendell-Rubin adjusted likelihood ratio test (LRT) was lastly evaluated, and where a nonsignificant value $(p>.05)$ occurs, this suggests that the additional class does not contribute statistically significantly to the description of the data. This analysis was conducted using Mplus version 7.11 (Muthén \& Muthén, 2013). Analysis of variance and chi-square tests were performed to assess differences in demographics and trauma history across the classes identified in the LCA.

Second, multinomial logistic regressions were conducted to assess childhood adversity in relation to class membership. Firstly, childhood adversity types were entered as predictor variables. In the second model, a cumulative childhood adversity variable was entered as a predictor variable. In both models, class membership was entered as a dependent variable, age and total adult trauma were included as a co-variates, and the following were entered as predictors; sex $(0=$ male, $1=$ female), marital status ( $0=$ not in committed relationship, $1=$ in a committed relationship), employment status $(0=$ unemployed, $1=$ employed $)$, education $(0=$ no university education, $1=$ university education), urbanicity $(0=$ rural, $1=$ urban), migrant status $(0=$ no migration, $1=$ migration) and cannabis use ( $0=$ never used cannabis, $1=$ used cannabis).

Third, the level of co-morbid psychopathology was investigated in relation to class membership. Two ANOVAs were conducted with total depression and total anxiety score entered as dependent variables and class membership entered as an independent variable. All descriptive analyses, regression analyses and analysis of variance were performed using the Statistical Package for Social Science (SPSS, version 23.0).

\section{Results}

The frequencies of PTSD, DSO and psychosis symptom-endorsement are presented in Table 1. The endorsement of traumatic stress symptoms significantly differed by sex; females were more likely to report all PTSD and DSO symptoms. The endorsement of two psychosis items differed by sex; men were more likely to report being "under control" or having "special powers". At the symptom level (i.e. psychosis experiences accompanied by distress) the endorsement of three psychosis symptoms differed by sex; men were more likely to report receiving "special messages", being "under control" and "having special powers".

The most commonly reported type of childhood adversity was "verbal or physical threats" (36.2\%) followed by "emotional neglect" (35.9\%), "physical assault" (34.2\%), "sexual assault" (16.1\%) and "physical neglect" (10.2\%). The mean number of trauma types reported was 1.32 $(S D=1.20$, range $=1-5)$. Regarding total adult trauma, the mean number of potentially traumatic events experienced was $2.14(\mathrm{SD}=2.46$, range $=1-17)$. 
Table 1. Frequency of symptom endorsement $(\mathrm{N}=1051)$.

\begin{tabular}{|c|c|c|c|c|}
\hline Sympton & & Male (\%) & Female (\%) & Total (\%) \\
\hline \multicolumn{5}{|l|}{ PTSD } \\
\hline & Re-experiencing & 28.0 & 40.6 & $36.6^{*}$ \\
\hline & Sense of threat & 31.6 & 47.0 & $42.2^{*}$ \\
\hline & Avoidance & 31.6 & 42.1 & $38.8^{*}$ \\
\hline \multicolumn{5}{|l|}{ DSO } \\
\hline & Affective dysregulation & 41.9 & 51.3 & $51.3^{*}$ \\
\hline & Negative self-concept & 34.6 & 41.4 & $39.3^{*}$ \\
\hline & Disturbed relationships & 42.5 & 47.4 & $47.4^{*}$ \\
\hline \multicolumn{5}{|c|}{ Psychosis: Frequency } \\
\hline & Mind reading & 23.8 & 21.8 & 22.5 \\
\hline & Special messages & 9.9 & 7.2 & 8.1 \\
\hline & Spying & 27.7 & 25.0 & 25.9 \\
\hline & Voice hearing & 20.8 & 19.9 & 20.2 \\
\hline & Under control & 14.5 & 9.0 & $10.8^{*}$ \\
\hline & Visual hallucination & 18.7 & 18.2 & 18.4 \\
\hline & Special powers & 16.3 & 11.4 & $12.9^{*}$ \\
\hline \multicolumn{5}{|c|}{ Psychosis: Distress } \\
\hline & Mind reading & 11.4 & 8.2 & 9.2 \\
\hline & Special messages & 7.8 & 3.3 & $4.8^{*}$ \\
\hline & Spying & 22.3 & 21.3 & 21.6 \\
\hline & Voice hearing & 14.5 & 12.5 & 13.1 \\
\hline & Under control & 11.4 & 5.7 & $7.5^{*}$ \\
\hline & Visual hallucination & 13.3 & 10.2 & 11.1 \\
\hline & Special powers & 9.9 & 4.7 & $6.4^{*}$ \\
\hline
\end{tabular}

Table 2. Fit statistics for the latent class analysis $(\mathrm{N}=1051)$.

\begin{tabular}{lcrrrrc}
\hline Classes & Log-likelihood & AIC & BIC & ssaBIC & LRT $(p)$ & Entropy \\
\hline 1 & -6645.803 & 13,317 & 13,382 & 13,340 & - & - \\
2 & -5265.824 & 10,585 & 10,719 & 10,633 & $2731.911(0.0000)$ & 0.890 \\
3 & -4851.397 & 9784 & 9988 & 9857 & $820.431(0.0000)$ & 0.891 \\
4 & -4768.612 & 9647 & 9919 & 9745 & $163.887(0.0000)$ & 0.847 \\
5 & -4695.963 & 9529 & 9871 & 9652 & $143.823(0.0006)$ & 0.860 \\
$\mathbf{6}$ & $-\mathbf{4 6 3 1 . 9 8 9}$ & $\mathbf{9 4 2 9}$ & $\mathbf{9 8 4 1}$ & $\mathbf{9 5 7 7}$ & $\mathbf{1 2 6 . 6 4 6 ( \mathbf { 0 . 0 0 0 1 ) }}$ & $\mathbf{0 . 8 2 2}$ \\
7 & $-4585.642^{*}$ & 9365 & 9846 & 9538 & $91.753(0.0108)$ & 0.836 \\
8 & $-4563.067^{*}$ & 9348 & 9898 & 9545 & $44.690(0.1908)$ & 0.846 \\
\hline
\end{tabular}

Note. AIC = Akaike Information Criterion; BIC = Bayesian Information Criterion; ssaBIC = sample size adjusted Bayesian Information Criterion; LRT = Lo-Mendell-Rubin adjusted likelihood ratio test. Best fitting LCA model in bold. ${ }^{*}=$ The best loglikelihood value was not replicated; The p-value may not be trustworthy due to local maxima.

The fit statistics for the LCA are presented in Table 2. The BIC identified model 6 as providing the best fit for the data. This finding was corroborated by the AIC, ssaBIC and the entropy-values. Upon inspection of the profile plot, the classes lent themselves to theoretically meaningful interpretation. The profile plot and probabilities for the six-class solution are shown in Figure 1.

Class 1 ( $n=204,19 \%)$ was characterized by elevated reports of PTSD and DSO. This class was labelled "CPTSD". Class 2 ( $n=434,41.3 \%$ ) was the largest class and was characterised by low probabilities of reporting any form of symptomatology. This class was labelled "low symptom". Class 3 ( $n=117,11.1 \%$ ) was characterized by elevated reports of PTSD symptomatology. This class was labelled "PTSD". Class $4(n=170,16.2 \%)$ was characterised by elevated risk of DSO. This class was labelled "DSO". Class 5 ( $n=90,8.6 \%$ ) was characterized by a moderate to high probability of reporting all PTSD and DSO symptoms but a varying probability for the 7 psychosis symptoms; a relatively lower probability of endorsing psychosis items 1, 2, 5 and 7 (mind reading, special messages, being under control and special powers) and a relatively higher probability of endorsing psychosis items 3, 4 and 6 (spying on you, auditory and visual hallucinations). This class was 


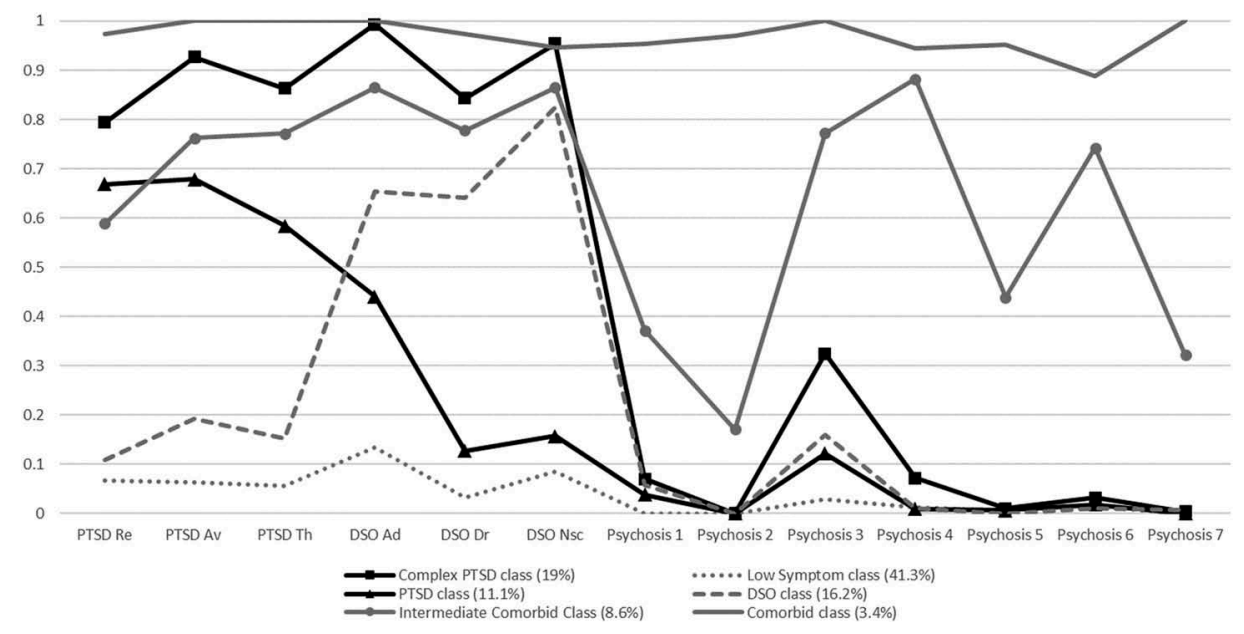

Figure 1. Symptom endorsement of PTSD, CPTSD and psychosis items by class.

Note. $\mathrm{Y}$-axis $=$ probability of symptom endorsement; $\mathrm{X}$-axis $=$ measured study symptoms; PTSD $=$ Post-Traumatic Stress Disorder; DSO = Disturbances in Self-Organisation; Re = Re-experiencing; $\mathrm{Av}=$ Avoidance; $\mathrm{Th}=$ Sense of threat; Ad = Affective dysregulation; $\mathrm{Dr}=$ Disturbances in relationships; Nsc $=$ Negative self-concept; See method section "psychosis symptoms" for description of psychosis items $1-7$.

labelled "intermediate comorbid". Finally, class 6 was the smallest class ( $\mathrm{n}=36,3.4 \%$ ) and was characterized by extremely high risk of endorsing PTSD, DSO and psychosis symptoms. This class was labelled "comorbid". The distribution of demographic and trauma exposure variables across the resultant classes are presented in Table 3.

Table 4 shows the results of the multinomial logistic regression analyses testing different types of childhood adversity as predictors of class membership. The model was statistically significant with the "low symptom" class used as the reference category $\left(X^{2}(70)=593.93, p<.001\right)$.

Table 5 shows the results of the model testing cumulative childhood adversity as a predictor of class membership. The model was also significant when the "low symptom" class was set as the reference category $\left(X^{2}(65)=565.79, p<.001\right)$. Total adult trauma scores and age significantly differed across the resultant classes. Classes characterised by traumatic stress symptoms and psychosis symptoms demonstrated the strongest dose-response effects.

Table 3. Demographic and trauma history characteristics of the classes.

\begin{tabular}{|c|c|c|c|c|c|c|}
\hline Characteristics & $\begin{array}{c}\text { Class 1: CPTSD }{ }^{\mathrm{a}} \\
\mathrm{n}=204\end{array}$ & $\begin{array}{l}\text { Class 2: Low } \\
n=434\end{array}$ & $\begin{array}{c}\text { Class 3: PTSD }{ }^{c} \\
n=117\end{array}$ & $\begin{array}{l}\text { Class 4: DSO }{ }^{d} \\
n=170\end{array}$ & $\begin{array}{l}\text { Class 5: Intermediate }{ }^{\mathrm{e}} \\
\mathrm{n}=90\end{array}$ & $\begin{array}{c}\text { Class 6: Comorbid }{ }^{f} \\
n=36\end{array}$ \\
\hline Sex (female) & $80.9 \%$ & $61.5 \%$ & $77.8 \%$ & $69.4 \%$ & $68.9 \%$ & $44.4 \%$ \\
\hline Urban status & $46.1 \%$ & $41.7 \%$ & $44.0 \%$ & $40.8 \%$ & $51.1 \%$ & $94.4 \%$ \\
\hline Relationship & $65.7 \%$ & $70.0 \%$ & $84.6 \%$ & $64.1 \%$ & $68.9 \%$ & $88.9 \%$ \\
\hline Migration & $19.6 \%$ & $16.1 \%$ & $14.5 \%$ & $17.6 \%$ & $16.7 \%$ & $41.7 \%$ \\
\hline Employed & $58.8 \%$ & $54.4 \%$ & $65.0 \%$ & $62.9 \%$ & $55.6 \%$ & $72.2 \%$ \\
\hline Education & $64.2 \%$ & $62.2 \%$ & $56.4 \%$ & $66.5 \%$ & $64.4 \%$ & $58.3 \%$ \\
\hline Cannabis use & $44.6 \%$ & $22.8 \%$ & $24.8 \%$ & $34.7 \%$ & $51.1 \%$ & $55.6 \%$ \\
\hline Verbal threats & $53.9 \%$ & $19.4 \%$ & $36.8 \%$ & $35.9 \%$ & $60.0 \%$ & $77.8 \%$ \\
\hline Emotional neglect & $55.9 \%$ & $16.1 \%$ & $35.0 \%$ & $44.7 \%$ & $57.8 \%$ & $66.7 \%$ \\
\hline Physical neglect & $16.2 \%$ & $3.5 \%$ & $5.1 \%$ & $7.1 \%$ & $28.9 \%$ & $41.7 \%$ \\
\hline Sexual abuse & $24.0 \%$ & $9.0 \%$ & $17.9 \%$ & $10.0 \%$ & $27.8 \%$ & $50.0 \%$ \\
\hline Physical abuse & $48.5 \%$ & $20.5 \%$ & $39.3 \%$ & $30.0 \%$ & $52.2 \%$ & $75.0 \%$ \\
\hline Mean Age & $41.63(13.20)$ & $53.28(14.77)^{\text {adef }}$ & $49.91(14.00)^{\text {adef }}$ & $43.22(13.22)$ & $39.32(12.34)$ & $34.50(9.41)$ \\
\hline Mean LEC total & $2.8(2.28)^{\mathrm{bd}}$ & $1.73(1.74)$ & $2.56(2.07)^{\mathrm{b}}$ & $1.95(1.57)$ & $3.77(3.24)^{\mathrm{abcd}}$ & $6.52(5.57)$ \\
\hline
\end{tabular}

Note. All chi-square tests were 5 degrees of freedom; LEC = Life Events Checklist for total adult trauma; ${ }^{\text {abcdef }}$ Indicates the classes from which the respective class-scores on clinical outcomes differs significantly; all post-hoc comparisons were conducted with Bonferroni correction. 
Table 4. Trauma history and demographic variables as predictors of class membership.

\begin{tabular}{lccccc}
\hline Characteristics & Class 1: CPTSD & Class 3: PTSD & Class 4: DSO & Class 5: Intermediate & $\begin{array}{c}\text { Class 6: Comorbid } \\
\text { OR }(95 \% \mathrm{Cl})\end{array}$ \\
\hline Sex (female) & $1.54(0.97-2.45)$ & $2.08(1.24-3.50)^{*}$ & $0.88(0.57-1.35)$ & $0.75(0.41-1.37)$ & $0.28(0.11-0.71)$ \\
Urban & $1.25(0.85-1.85)$ & $1.16(0.75-1.79)$ & $0.99(0.67-1.45)$ & $1.32(0.78-2.23)$ & $15.23(3.30-70.24)^{*}$ \\
Relationship & $0.74(0.49-1.12)$ & $2.50(1.41-4.43)^{*}$ & $0.70(0.47-1.06)$ & $0.83(0.47-1.46)$ & $2.46(0.73-8.28)$ \\
Migration & $1.02(0.61-1.71)$ & $0.86(0.46-1.59)$ & $1.14(0.68-1.91)$ & $0.66(0.32-1.37)$ & $1.22(0.43-3.44)$ \\
Employed & $0.88(0.59-1.32)$ & $1.48(0.93-2.35)$ & $1.04(0.70-1.55)$ & $0.69(0.41-1.17)$ & $0.98(0.38-2.51)$ \\
Education & $0.72(0.47-1.08)$ & $0.66(0.42-1.04)$ & $0.84(0.55-1.27)$ & $0.77(0.44-1.34)$ & $0.78(0.31-1.94)$ \\
Cannabis use & $1.74(1.15-2.62)^{*}$ & $0.90(0.54-1.50)$ & $1.35(0.89-2.06)$ & $1.95(1.14-3.33)^{*}$ & $1.25(0.51-3.08)$ \\
Verbal threats & $1.85(1.08-3.16)^{*}$ & $1.36(0.73-2.54)$ & $1.32(0.76-2.30)$ & $2.36(1.15-4.80)^{*}$ & $2.28(0.64-8.12)$ \\
Emotional neglect & $3.22(1.08-3.16)^{*}$ & $1.76(1.02-3.06)^{*}$ & $3.66(2.28-5.89)^{*}$ & $2.54(1.35-4.79)^{*}$ & $2.54(0.87-7.42)$ \\
Physical neglect & $1.32(0.62-2.82)$ & $0.54(0.19-1.55)$ & $0.84(0.35-2.04)$ & $2.66(1.35-6.25)^{*}$ & $0.93(0.25-3.53)$ \\
Sexual abuse & $1.84(1.06-3.20)^{*}$ & $1.71(0.92-3.18)$ & $0.90(0.47-1.72)$ & $1.94(0.97-3.89)$ & $2.72(0.94-7.89)$ \\
Physical abuse & $1.18(0.70-1.99)$ & $1.61(0.88-2.92)$ & $0.81(0.47-1.40)$ & $1.02(0.51-2.06)$ & $2.42(0.71-8.18)$ \\
\hline
\end{tabular}

Note. The "low symptom" class was set as the baseline category; OR $(95 \% \mathrm{Cl})=$ Odds Ratio with $95 \%$ confidence interval.

${ }^{*} p<0.05$.

Table 5. Cumulative childhood adversity as predictor of class membership.

\begin{tabular}{lccccc}
\hline Trauma count & $\begin{array}{c}\text { Class 1: CPTSD } \\
\text { OR }(95 \% \mathrm{Cl})\end{array}$ & $\begin{array}{c}\text { Class 3: PTSD } \\
\text { OR }(95 \% \mathrm{Cl})\end{array}$ & $\begin{array}{c}\text { Class 4: DSO } \\
\text { OR }(95 \% \mathrm{Cl})\end{array}$ & $\begin{array}{c}\text { Class 5: Intermediate } \\
\text { OR }(95 \% \mathrm{Cl})\end{array}$ & $\begin{array}{c}\text { Class 6: Comorbid } \\
\text { OR }(95 \% \mathrm{Cl})\end{array}$ \\
\hline None & $\operatorname{Ref}$ & $\operatorname{Ref}$ & $\operatorname{Ref}$ & Ref & Ref \\
One & $2.78(1.62-4.79)^{*}$ & $1.43(0.78-2.62)$ & $1.49(0.89-2.51)$ & $1.89(0.83-4.30)$ & $4.00(0.87-18.53)$ \\
Two & $4.86(2.76-8.57)^{*}$ & $2.49(1.32-4.69)^{*}$ & $2.39(1.37-4.16)^{*}$ & $4.42(2.04-9.56)^{*}$ & $5.77(1.19-27.92)^{*}$ \\
Three & $7.58(4.10-14.01)^{*}$ & $3.05(1.51-6.17)^{*}$ & $3.44(1.86-6.36)^{*}$ & $6.65(2.96-14.93)^{*}$ & $11.71(2.85-48.14)^{*}$ \\
Four or more & $10.69(5.03-22.69)^{*}$ & $3.41(1.38-8.41)^{*}$ & $2.51(1.03-6.09)^{*}$ & $15.34(6.31-37.25)^{*}$ & $24.74(5.90-103.77)^{*}$ \\
\hline
\end{tabular}

Note. The "low symptom" class was set as the baseline category; None; zero childhood adversity's was set as the reference trauma category; covariates controlled for in this model included age, total adult trauma, sex (female), urban status, relationship status, migration status, employment, education level, and cannabis use. ${ }^{*} p<0.05$.

Table 6. Total depression and total anxiety scores across the latent classes.

\begin{tabular}{lcccccc}
\hline & ${\text { Class 1: } \text { CPTSD }^{\mathrm{a}}}$ & Class 2: Low $^{\mathrm{b}}$ & Class 3: PTSD $^{\mathrm{c}}$ & Class 4: DSO $^{\mathrm{d}}$ & Class 5: Intermediate $^{\mathrm{e}}$ & Class 6: Comorbid $^{\mathrm{f}}$ \\
Total score & $\mathrm{n}=204$ & $\mathrm{n}=434$ & $\mathrm{n}=117$ & $\mathrm{n}=170$ & $\mathrm{n}=90$ & $\mathrm{n}=36$ \\
\hline Depression & $24.10(6.72)^{\mathrm{bcd}}$ & $11.43(3.21)^{\text {acdef }}$ & $14.56(4.76)$ & $18.80(6.31)^{\mathrm{c}}$ & $24.58(7.20)^{\mathrm{bcd}}$ & $24.92(7.65)^{\mathrm{bcd}}$ \\
Anxiety & $19.39(5.82)^{\mathrm{bcd}}$ & $8.77(2.78)^{\text {acdef }}$ & $11.78(4.45)$ & $14.52(5.41)^{\mathrm{c}}$ & $19.60(6.08)^{\mathrm{bcd}}$ & $20.00(5.52)^{\mathrm{bcd}}$ \\
\hline
\end{tabular}

Note. Each class has been ascribed a letter, the presence of ${ }^{\text {abcdef }}$ indicates the classes from which the respective class-scores on clinical outcomes differs significantly; all post hoc comparisons were conducted with Bonferroni correction.

A series of ANOVAs were conducted to investigate differences in depression and anxiety across classes. Total depression score significantly differed across class, $F(5,1045)=233.73, p<.001$, as did total anxiety score, $F(5,1045)=212.25, p<.001)$. The adjusted means and standard deviations for both tests are presented in Table 6. Post hoc comparisons revealed that for both depression and anxiety, participants in the "CPTSD" class, "Intermediate comorbid" class, as well as the "Comorbid" class all scored significantly higher than participants in the "PTSD" class, "DSO" class and "Low symptom" class. Furthermore, participants in the "DSO" class scored significantly higher than participants in the "PTSD" class, who both scored significantly higher than participants in the "Low symptom" group.

\section{Discussion}

This study evaluated the co-occurrence of psychosis symptoms and ICD-11 traumatic stress symptoms among a trauma-exposed UK adult population sample. Six classes emerged: Separate PTSD and CPTSD classes consistent with ICD-11 diagnostic formulations (Brewin et al., 2017; Cloitre et al., 2018; Maercker et al., 2013). Two classes were characterised by co-occurring psychosis and traumatic stress symptoms; a "comorbid" class denoted by a high probability of endorsing all 
psychosis symptoms and CPTSD symptoms; as well as an "intermediate comorbid" class denoted by a moderate probability of endorsing PTSD and DSO symptoms but a varying probability of endorsing psychosis symptoms. Finally, a "DSO" class characterised by a moderate to high probability of endorsing only DSO symptoms, and a "low symptom" class characterised by a low probability of endorsing all symptoms.

Notably, all classes with a high probability of endorsing psychosis symptoms were also characterised by traumatic stress symptoms. This finding is consistent with a previous LCA study in which psychosis symptoms were part of a broader clinical picture including symptoms of posttraumatic stress (Shevlin et al., 2011). Psychosis and PTSD have been conceptualised as similar entities that lie on a spectrum of reactions to trauma (Morrison et al., 2003, 2005) and empirical reports support the co-occurrence of traumatic stress and psychosis symptoms with relatively high rates of PTSD/psychotic disorder comorbidity (Achim et al., 2009; Kessler et al., 2005). The current study extends this co-occurrence to symptoms of ICD-11 CPTSD. If these findings are representative of trauma-exposed populations, high comorbidity should be expected between psychosis and ICD11 traumatic stress diagnoses in the general population. Previous studies have alluded to a categorical interpretation of the relationship between the symptoms, suggesting that there may be a psychotic PTSD subtype (Braakman, Kortmann, \& Van Den Brink, 2009). While it is important to recognise psychosis symptoms among traumatised groups, an alternative interpretation of the findings is that the qualitative distinction between the classes reflect different levels of severity of posttraumatic sequelae along an underlying continuum.

An increasing number of childhood maltreatment types increased the risk of membership to the PTSD, CPTSD, intermediate and comorbid classes in a distinct dose-response manner. This finding is consistent with previous research investigating key risk factors for psychosis and traumatic stress constructs in isolation (Cloitre et al., 2013; Karatzias et al., 2017; Shevlin et al., 2008; Trauelsen et al., 2015). The dose-response effect was strongest for the comorbid classes. The combined effect of multiple interpersonal traumas has been linked to an elevated vulnerability towards "symptom complexity" - a concept which refers to several simultaneously presenting but discrete psychological difficulties (Briere \& Scott, 2015). This finding could support the notion that there may be a trauma spectrum ranging from PTSD and CPTSD to psychosis. The cumulative effect of childhood adversity may be stronger for those who endorse elevated levels of all psychopathology as certain psychological processes, for example emotion regulation - which has been linked to trauma exposure, traumatic stress and psychosis (Hardy, 2017) - may be more impaired leaving an individual more vulnerable to developing multiple forms of psychopathology. Regarding specific types of child maltreatment, significant associations were observed but these did not readily differentiate between the resultant classes. Overall, the findings provide evidence that early life trauma poses a shared risk factor for psychosis, PTSD and CPTSD symptoms alike.

The predictive effect of early life trauma on class membership persisted despite controlling for total adult trauma as well as potential covariates including cannabis use, ethnicity, urbanicity and educational attainment (Houston et al., 2008; Shevlin et al., 2008). Cannabis use significantly increased the likelihood of membership to both the intermediate comorbid class and the CPTSD class. Urbanicity was the only variable that readily distinguished between the resultant classes, risk of membership to the comorbid class as compared to the low symptom class was fifteen times greater if an individual endorsed urban status. Urbanicity is a key etiological predictor of psychosis, a host of social factors mediate this association such as neighbourhood composition, deprivation and social fragmentation (Vassos, Pedersen, Murray, Collier, \& Lewis, 2012). The CPTSD, the intermediate comorbid and the comorbid classes reported significantly higher depression and anxiety scores compared to the low symptom, the DSO and the PTSD classes. It is possible that CPTSD is a particularly debilitating disorder associated with relatively high levels of psychiatric comorbidity regardless of the presence or absence of psychosis symptoms. However, further research is necessary to replicate these findings. 
The study had several limitations. First, given the cross-sectional nature of the data it is not possible to determine the temporal ordering of either psychopathology, nor is it possible to establish causality for early life trauma and adult mental health outcomes. Moreover, all symptoms were measured via self-report assessment. Second, the measurement of psychosis symptoms can be confounded by respondents misunderstanding or normalising psychosis experiences (Shevlin et al., 2011). Given that this was a general population sample, there is also a risk that psychosis experiences were underreported due to stigma associated with psychosis (Bebbington \& Kuipers, 1994). Third, although the sample was drawn from a nationally representative cohort, females and individuals who have completed college or university education are overly represented. Finally, the question on migration was worded, "Have you ever left one country to live in another country?" so there may be ambiguity about whether a positive response indicated that the respondent had migrated to the UK or if they had temporarily moved and lived in another country and then returned to the UK.

The current study provides further evidence that a subgroup of trauma-exposed individuals exhibited psychosis symptoms alongside traumatic stress symptoms, no group of participants was identified that displayed psychosis symptoms without also reporting symptoms of posttraumatic stress. This finding has important clinical implications: If psychosis symptoms have a high probability of emerging in the context of early life trauma and traumatic stress, it needs to be established if existing trauma treatments are suitable for targeting these symptoms or whether new trauma treatments should be developed to target psychosis symptoms. In terms of further research, it might also be worthwhile to explore ICD-11 PTSD and CPTSD in a population with a primary diagnosis of a schizophrenia spectrum disorders.

\section{Disclosure statement}

No potential conflict of interest was reported by the authors.

\section{ORCID}

Rachel Frost (iD http://orcid.org/0000-0002-5078-171X

Maria Louison Vang (iD http://orcid.org/0000-0001-7031-4070

Thanos Karatzias (iD) http://orcid.org/0000-0002-3002-0630

Philip Hyland (iD http://orcid.org/0000-0002-9574-7128

Mark Shevlin (iD http://orcid.org/0000-0001-6262-5223

\section{References}

Achim, A. M., Maziade, M., Raymond, É., Olivier, D., Mérette, C., \& Roy, M.-A. (2009). How prevalent are anxiety disorders in schizophrenia? A meta-analysis and critical review on a significant association. Schizophrenia Bulletin, 37(4), 811-821.

Akaike, H. (1998). Factor analysis and AIC. In E. Parzen, K. Tanabe, \& G. Kitagawa (Eds.), Selected papers of hirotugu akaike (pp. 371-386). New York: Springer New York.

Arseneault, L., Cannon, M., Fisher, H. L., Polanczyk, G., Moffitt, T. E., \& Caspi, A. (2011). Childhood trauma and children's emerging psychotic symptoms: A genetically sensitive longitudinal cohort study. American Journal of Psychiatry, 168(1), 65-72.

Bailey, T., Alvarez-Jimenez, M., Garcia-Sanchez, A. M., Hulbert, C., Barlow, E., \& Bendall, S. (2018). Childhood trauma is associated with severity of hallucinations and delusions in psychotic disorders: A systematic review and meta-analysis. Schizophrenia Bulletin, 44(5), 1111-1122.

Bebbington, P., \& Kuipers, L. (1994). The social management of long standing schizophrenia I. The deployment of service resources. Clinician, 12, 17-29.

Braakman, M. H., Kortmann, F. A. M., \& Van Den Brink, W. (2009). Validity of 'post-traumatic stress disorder with secondary psychotic features': A review of the evidence. Acta Psychiatrica Scandinavica, 119(1), 15-24. 
Brewin, C. R., Cloitre, M., Hyland, P., Shevlin, M., Maercker, A., Bryant, R. A., ... Rousseau, C. (2017). A review of current evidence regarding the ICD-11 proposals for diagnosing PTSD and complex PTSD. Clinical Psychology Review, 58, $1-15$.

Briere, J., \& Scott, C. (2015). Complex trauma in adolescents and adults: Effects and treatment. Psychiatric Clinics, 38(3), 515-527.

Cloitre, M., Garvert, D. W., Brewin, C. R., Bryant, R. A., \& Maercker, A. (2013). Evidence for proposed ICD-11 PTSD and complex PTSD: A latent profile analysis. European Journal of Psychotraumatology, 4(1), 20706.

Cloitre, M., Shevlin, M., Brewin, C. R., Bisson, J. I., Roberts, N. P., Maercker, A., ... Hyland, P. (2018). The International Trauma Questionnaire: Development of a self-report measure of ICD-11 PTSD and complex PTSD. Acta Psychiatrica Scandinavica, 138(6), 536-546.

Felitti, V. J., Anda, R. F., Nordenberg, D., Williamson, D. F., Spitz, A. M., Edwards, V., ... Marks, J. S. (1998). Relationship of childhood abuse and household dysfunction to many of the leading causes of death in adults: The Adverse Childhood Experiences (ACE) study. American Journal of Preventive Medicine, 14(4), 245-258.

Gibson, L. E., Alloy, L. B., \& Ellman, L. M. (2016). Trauma and the psychosis spectrum: A review of symptom specificity and explanatory mechanisms. Clinical Psychology Review, 49, 92-105.

Hardy, K. V. (2017). Pathways from trauma to psychotic experiences: A theoretically informed model of posttraumatic stress in psychosis. Frontiers in Psychology, 8, 697.

Hardy, K. V., \& Mueser, K. T. (2017). Editorial: Trauma, psychosis and posttraumatic stress disorder. Frontiers in Psychiatry, 8(220). doi:10.3389/fpsyt.2017.00220

Herman, J. L. (1992). Complex PTSD: A syndrome in survivors of prolonged and repeated trauma. Journal of Traumatic Stress, 5(3), 377-391.

Houston, J. E., Murphy, J., Adamson, G., Stringer, M., \& Shevlin, M. (2008). Childhood sexual abuse, early cannabis use, and psychosis: Testing an interaction model based on the national comorbidity survey. Schizophrenia Bulletin, 34(3), 580-585.

Husted, J. A., Ahmed, R., Chow, E. W. C., Brzustowicz, L. M., \& Bassett, A. S. (2010). Childhood trauma and genetic factors in familial schizophrenia associated with the NOS1AP gene. Schizophrenia Research, 121(1), 187-192.

Karatzias, T., Shevlin, M., Fyvie, C., Hyland, P., Efthymiadou, E., Wilson, D., ... Cloitre, M. (2017). Evidence of distinct profiles of Posttraumatic Stress Disorder (PTSD) and Complex Posttraumatic Stress Disorder (CPTSD) based on the new ICD-11 Trauma Questionnaire (ICD-TQ). Journal of Affective Disorders, 207, 181-187.

Kelleher, I., Harley, M., Murtagh, A., \& Cannon, M. (2011). Are screening instruments valid for psychotic-like experiences? A validation study of screening questions for psychotic-like experiences using in-depth clinical interview. Schizophrenia Bulletin, 37(2), 362-369.

Kessler, R. C., Berglund, P., Demler, O., Jin, R., Merikangas, K. R., \& Walters, E. E. (2005). Lifetime prevalence and age-ofonset distributions of DSM-IV disorders in the national comorbidity survey replication. Archives of General Psychiatry, 62(6), 593-602.

Kroenke, K., Spitzer, R. L., \& Williams, J. B. W. (2001). The PHQ-9. Journal of General Internal Medicine, 16(9), 606-613.

Maercker, A., Brewin, C. R., Bryant, R. A., Cloitre, M., Reed, G. M., van Ommeren, M., ... Llosa, A. E. (2013). Proposals for mental disorders specifically associated with stress in the international classification of diseases-11. The Lancet, 381 (9878), 1683-1685.

Morrison, A., Read, J., \& Turkington, D. (2005). Trauma and psychosis: Theoretical and clinical implications. Acta Psychiatrica Scandinavica, 112(5), 327-329.

Morrison, A. P., Frame, L., \& Larkin, W. (2003). Relationships between trauma and psychosis: A review and integration. British Journal of Clinical Psychology, 42(4), 331-353.

Muthén, L. K., \& Muthén, B. O. (2013). Mplus (Version 7.11). Los Angeles, CA: Muthén \& Muthén.

Nolan, E., Murphy, S., O'Neill, T., Houston, J., Murphy, J., \& Shevlin, M. (2018). Prevalence of psychotic-like experiences and associated distress in adolescent community, sexual-trauma and clinical samples. Psychosis; Psychological, Social and Integrative Approaches, 10(4), 251-262.

Nylund, K. L., Asparouhov, T., \& Muthén, B. O. (2007). Deciding on the number of classes in latent class analysis and growth mixture modeling: A monte carlo simulation study AU - Nylund, Karen L. Structural Equation Modeling: A Multidisciplinary Journal, 14(4), 535-569.

Read, J., van Os, J., Morrison, A. P., \& Ross, C. A. (2005). Childhood trauma, psychosis and schizophrenia: A literature review with theoretical and clinical implications. Acta Psychiatrica Scandinavica, 112(5), 330-350.

Schäfer, I., \& Fisher, H. L. (2011). Childhood trauma and psychosis - what is the evidence? Dialogues in Clinical Neuroscience, 13(3), 360-365.

Schwarz, G. (1978). Estimating the dimension of a model. Annals of Statistics, 6(2), 461-464.

Sclove, S. L. (1987). Application of model-selection criteria to some problems in multivariate analysis. Psychometrika, 52 (3), 333-343.

Shevlin, M., Armour, C., Murphy, J., Houston, J. E., \& Adamson, G. (2011). Evidence for a psychotic posttraumatic stress disorder subtype based on the national comorbidity survey. Social Psychiatry and Psychiatric Epidemiology, 46(11), 1069-1078. 
Shevlin, M., Houston, J. E., Dorahy, M. J., \& Adamson, G. (2008). Cumulative traumas and psychosis: An analysis of the national comorbidity survey and the british psychiatric morbidity survey. Schizophrenia Bulletin, 34(1), $193-199$.

Spitzer, R. L., Kroenke, K., Williams, J. B. W., \& Löwe, B. (2006). A brief measure for assessing generalized anxiety disorder: The GAD-7. Archives of Internal Medicine, 166(10), 1092-1097.

Trauelsen, A. M., Bendall, S., Jansen, J. E., Nielsen, H.-G. L., Pedersen, M. B., Trier, C. H., ... Simonsen, E. (2015). Childhood adversity specificity and dose-response effect in non-affective first-episode psychosis. Schizophrenia Research, 165(1), 52-59.

Varese, F., Smeets, F., Drukker, M., Lieverse, R., Lataster, T., Viechtbauer, W., .. Bentall, R. P. (2012). Childhood adversities increase the risk of psychosis: A meta-analysis of patient-control, prospective- and cross-sectional cohort studies. Schizophrenia Bulletin, 38(4), 661-671.

Vassos, E., Pedersen, C. B., Murray, R. M., Collier, D. A., \& Lewis, C. M. (2012). Meta-analysis of the association of urbanicity with schizophrenia. Schizophrenia Bulletin, 38(6), 1118-1123.

Weathers, F. W., Blake, D. D., Schnurr, P. P., Kaloupek, D. G., Marx, B. P., \& Keane, T. M. (2013). The life events checklist for DSM-5 (LEC-5). Instrument available from the National Center for PTSD. Retrieved from www.ptsd.va.gov

Yuan, K. H., \& Bentler, P. M. (2000). Three likelihood-based methods for mean and covariance structure analysis with nonnormal missing data. Sociological Methodology, 30(1), 165-200. 